\title{
Amygdala and Ventromedial Prefrontal Cortex Are Inversely Coupled during Regulation of Negative Affect and Predict the Diurnal Pattern of Cortisol Secretion among Older Adults
}

\author{
Heather L. Urry, ${ }^{1,2}$ Carien M. van Reekum, ${ }^{1,2}$ Tom Johnstone, ${ }^{1}$ Ned H. Kalin, ${ }^{2,3}$ Marchell E. Thurow, ${ }^{2}$ Hillary S. Schaefer, ${ }^{1}$ \\ Cory A. Jackson, ${ }^{2}$ Corrina J. Frye, ${ }^{2}$ Lawrence L. Greischar, ${ }^{2}$ Andrew L. Alexander, ${ }^{2,3,4}$ and Richard J. Davidson ${ }^{1,2,3}$ \\ ${ }^{1}$ Waisman Laboratory for Brain Imaging and Behavior and Departments of ${ }^{2}$ Psychology, ${ }^{3}$ Psychiatry, and ${ }^{4}$ Medical Physics, University of \\ Wisconsin-Madison, Madison, Wisconsin 53706
}

\begin{abstract}
Among younger adults, the ability to willfully regulate negative affect, enabling effective responses to stressful experiences, engages regions of prefrontal cortex (PFC) and the amygdala. Because regions of PFC and the amygdala are known to influence the hypothalamicpituitary-adrenal axis, here we test whether PFC and amygdala responses during emotion regulation predict the diurnal pattern of salivary cortisol secretion. We also test whether PFC and amygdala regions are engaged during emotion regulation in older (62- to 64-year-old) rather than younger individuals. We measured brain activity using functional magnetic resonance imaging as participants regulated (increased or decreased) their affective responses or attended to negative picture stimuli. We also collected saliva samples for 1 week at home for cortisol assay. Consistent with previous work in younger samples, increasing negative affect resulted in ventral lateral, dorsolateral, and dorsomedial regions of PFC and amygdala activation. In contrast to previous work, decreasing negative affect did not produce the predicted robust pattern of higher PFC and lower amygdala activation. Individuals demonstrating the predicted effect (decrease $<$ attend in the amygdala), however, exhibited higher signal in ventromedial prefrontal cortex (VMPFC) for the same contrast. Furthermore, participants displaying higher VMPFC and lower amygdala signal when decreasing compared with the attention control condition evidenced steeper, more normative declines in cortisol over the course of the day. Individual differences yielded the predicted link between brain function while reducing negative affect in the laboratory and diurnal regulation of endocrine activity in the home environment.
\end{abstract}

Key words: emotion regulation; amygdala; ventromedial prefrontal cortex; cortisol; negative affect; pupil dilation

\section{Introduction}

Responding adaptively to stressful experiences is facilitated by the ability to regulate negative emotion. Previous work in younger samples indicates that deliberately diminishing negative affective responses yields increased activation in lateral and dorsal regions of prefrontal cortex (PFC) and/or decreased activation in the amygdala (Beauregard et al., 2001; Ochsner et al., 2002, 2004; Schaefer et al., 2002; Levesque et al., 2003, 2004; Kalisch et al., 2005; Phan et al., 2005; Harenski and Hamann, 2006; Ohira et al., 2006), a pattern suggesting that PFC projections to the amygdala exert a top-down, inhibitory influence. The absence of direct connections between lateral/dorsal PFC and the amygdala, how-

\footnotetext{
Received Aug. 2, 2005; revised March 15, 2006; accepted March 20, 2006.

This work was supported by National Institutes of Mental Health Grants P50-MH61083, P50-MH52354, P50MH069315, and R37-MH43454, National Institute on Aging Grant P01-AG021079, and General Clinical Research Centers Program of the National Center for Research Resources, National Institutes of Health Grant M01-RR03186. We gratefully acknowledge the following individuals: Daren C. Jackson and Alexander J. Shackman for helpfu consultation, Michael Anderle and Ron Fisher for assistance with collection of the imaging data, Kristin Rae Evans for conducting the salivary cortisol assays, Joseph A. Hampel and Ann K. Chamberlain for assistance with cortiso preprocessing, and two anonymous reviewers for their very insightful comments.

Correspondence should be addressed to Heather L. Urry at her present address: Department of Psychology, Tufts University, 490 Boston Avenue, Medford, MA 02155. E-mail: heather.urry@tufts.edu.

D01:10.1523/JNEUROSCI.3215-05.2006

Copyright $\odot 2006$ Society for Neuroscience $\quad$ 0270-6474/06/264415-11\$15.00/0
}

ever, indicates that this apparently inhibitory effect must occur indirectly. One plausible route is via medial PFC (MPFC), which has direct connections with lateral/dorsal PFC and also with the amygdala (Price, 2005), and has been implicated in extinction of conditioned fear (Quirk et al., 2003; Phelps et al., 2004).

The amygdala projects to the hypothalamus, a set of nuclei that induces secretion of cortisol from the adrenal cortex by virtue of a hormonal cascade along the hypothalamic-pituitaryadrenal (HPA) axis (Sullivan et al., 2004). Cortisol is a hormone that stimulates glucose production and is therefore secreted in abundance in situations requiring mobilization of energy. Transient increases in circulating cortisol are superimposed on a normatively steep morning-to-evening diurnal rhythm in which the highest levels of cortisol are observed in the early morning hours, reaching their nadir in the evening (Baum and Grunberg, 1995). Flattened cortisol slopes are associated with stressful life circumstances (Polk et al., 2005; Ranjit et al., 2005) and with larger waist circumference, lower levels of social support, higher levels of perceived stress, and poorer explicit memory performance in healthy individuals (Abercrombie et al., 2004). In women with metastatic breast cancer, flatter slopes are associated with higher mortality rates (Sephton et al., 2000). Synthesizing these findings, one pathway leading to flattened cortisol slopes may be the failure to 
effectively regulate one's emotional responses, a possibility we investigate in the current report.

Older individuals were recruited for this effort from an ongoing longitudinal study focused on determining whether regulation-induced changes in brain function predict health and well-being in subsequent longitudinal follow-ups. They used cognitive reappraisal to amplify or attenuate their responses to unpleasant photographs in an event-related functional magnetic resonance imaging (fMRI) protocol during which pupil diameter was recorded to index cognitive resource allocation. In addition, participants collected saliva samples for 1 week at home to characterize each person's diurnal slope of cortisol secretion. We made four primary predictions: (1) replicating previous studies, deliberate attempts to increase and decrease negative affect experience should produce increased lateral/dorsal PFC activation; (2) whereas decreasing negative affect should produce decreased amygdala activation, increasing negative affect should produce increased amygdala activation; (3) MPFC and amygdala responses should be inversely associated during negative affect reduction; and (4) individuals exhibiting the steepest declines in cortisol from morning to night should also exhibit the biggest changes in PFC and the amygdala, a pattern suggesting adaptive HPA function in the face of effective emotion regulation.

\section{Materials and Methods \\ Participants}

Our sample of $n=19$ participants ( 11 females) was recruited from a larger sample of 84 right-handed respondents of the Wisconsin Longitudinal Study (WLS) who participated in a previous study in our laboratory (Jackson et al., 2003; Rosenkranz et al., 2003; Urry et al., 2004). The WLS is a longstanding effort to comprehensively describe the sociodemographic and psychological trajectories of $\sim 10,000$ men and women who were Wisconsin high school seniors in 1957 (for more information, see http://www.ssc.wisc.edu/ wls). Participants in the WLS, including participants in the present study, will continue to be followed in subsequent years, thus providing the opportunity to determine whether emotion regulation-related brain function at this stage predicts health and wellbeing later in life. Ages ranged from 62 to 64 years.

Participants reported no history of neurological issues or contraindications for MRI scanning (e.g., implanted cardiac or ferrous metal devices). In addition, using a brief screen of cognitive status, the Neurobehavioral Cognitive Status Examination (Northern California Neurobehavioral Group, Fairfax, CA), participants displayed intact performance relative to norms for healthy individuals ages 60-64 years across scales assessing attention, memory, comprehension, reasoning, and judgment (mean \pm SD throughout, $80.05 \pm 2.33$ ) (Macaulay et al., 2003). Although psychiatric interviews were not conducted, participants fell well within normal limits on questionnaire measures of depressive [Beck Depression Inventory-II (BDI) (Beck et al., 1996)] (3.09 \pm 4.11 ) and anxious [Trait version of the State-Trait Anxiety Inventory, Form X (STAI) (Spielberger et al., 1983)] (31.41 \pm 7.43$)$ symptomatology, the two most common forms of psychopathology. [In geriatric samples, a cutoff score of 8 is recommended for the BDI (Kim et al., 2002), whereas a cutoff of $54-55$ (determined using the State form) is recommended for the STAI (Kvaal et al., 2005).] Data from two participants (females) were excluded because of excessive movement during the scan, leaving us a final analytic sample size of $n=17$ (9 females) for the MR data, with a mean \pm SD age of $62.9 \pm 0.4$ years. Because of technical problems, pupil diameter data were unavailable for an additional five subjects, thus $n=$ 14 ( 8 females) for pupil analyses ( $n=12$ subjects providing both pupil and $\mathrm{fMRI}$ data). Informed consent was obtained and the rights of participants were protected according to the procedures set forth by the University of Wisconsin-Madison Health Sciences Institutional Review Board. Participants received $\$ 70.00$ for their participation in the MRI session.

\section{Stimulus materials}

We used a randomized event-related paradigm in which we presented one of two sets of digital color photographs $(800 \times 600$ resolution $)$ counterbalanced across participants in the scanner. The photographs were selected from the International Affective Picture System (IAPS) (Center for the Study of Emotion and Attention, 1999). Across the two sets, 72 negative photos were selected according to the IAPS norms to be both highly unpleasant $[1$, most unpleasant to 9 , most pleasant $(2.35 \pm$ $0.57)]$ and arousing $[1$, least arousing to 9 , most arousing $(5.82 \pm 0.80)]$, whereas 24 neutral photos were selected to be neither pleasant nor unpleasant $(5.04 \pm 0.29)$ and nonarousing $(3.14 \pm 0.79)$. In both sets, the negative pictures were significantly more unpleasant and arousing than the neutral pictures according to the normative ratings (all $p$ values $<0.05$ ). Picture presentation order was pseudorandomized with the constraint that no more than five pictures of the same valence were shown consecutively. Stimulus presentation was accomplished using the E-Prime software suite (Psychology Software Tools, Pittsburgh, PA) while visual stimulation was delivered via a fiber-optic goggle system (Avotec, Stuart, FL) interfaced with an eye-tracking system (SensoMotoric Instruments, Needham, MA) to measure pupil dilation during the scan session.

\section{Emotion regulation task}

Using a variant of the paradigm validated in our previous published electrophysiological work (Jackson et al., 2000), participants were trained to follow one of three instructions during each picture trial: "increase," "decrease," or "attend." The instruction to increase, presented via pneumatic headphones (Avotec), served as a cue to amplify the intensity of their negative affect (subjects heard the word "enhance"). For this task, participants were trained to either (1) imagine themselves or a loved one experiencing the situation being depicted, or (2) imagine a more extreme outcome than the one depicted (e.g., in response to a picture of a ferocious dog, a participant might imagine that the dog's leash broke and so the dog is about to bite them). Conversely, the instruction to decrease signified the cue to reduce the intensity of their negative affect, for which they were trained to either (1) view the situation as fake or unreal, or (2) imagine that the situation being depicted had a different outcome than the one suggested (e.g., victims of a car accident survived and healed well) (subjects heard the word "suppress"). Alternatively, on attend trials, participants were instructed to maintain their attention to the picture without changing their negative affective experience (subjects heard the word "maintain"). Presentation of regulation conditions was pseudorandomized such that no more than three trials of the same regulation condition were shown consecutively.

\section{Procedure}

Participants completed the laboratory portion of this experiment at the University of Wisconsin-Madison in two sessions separated by $1 \mathrm{~d}$. At the first session, participants underwent a "mock" scan to become acclimated to the sights, sounds, and procedures of the scanning environment and to train them to complete the regulation task using a standard set of instructions presented via an E-Prime stimulus presentation program. Participants were positioned inside the bore of an inactive MRI scanner shell, complete with bed, head coil, response box, and goggles, so they could practice completing a block of 24 trials of the regulation task. Simulated scanner sounds and task instructions were piped through earbud headphones. The practice block was succeeded by follow-up queries to determine whether participants were using the strategies as instructed and to shape as necessary.

The actual MRI scan session always occurred from 10:00 A.M. to 12:00 P.M. on the morning after the mock session. The timing was held constant in this manner so that associations between fMRI signal and salivary cortisol could not be attributed to variation in the time of day. This scan session was divided into four blocks of 24 trials. Before the real scan, the experimenter presented the task instructions again to consolidate participant understanding of their duties in the scanner. Anatomical images were collected after the functional runs. 


\section{Trial structure}

At the beginning of each $17 \mathrm{~s}$ trial, a white fixation cross was presented in the center of a black screen for $1 \mathrm{~s}$. This was coupled with a simultaneous tone to ensure visual attention to the impending trial. The fixation cross/ tone combination was followed by presentation of a picture for $11 \mathrm{~s}$. The regulation instruction (increase, attend, or decrease) was delivered at 3 , 4,5 , or $6 \mathrm{~s}$ after picture onset, a design feature that provided time for unregulated affect to be elicited before applying voluntary regulatory strategies. The variation in time of instruction onset allowed us to uniquely partition variance associated with regulation of negative emotion at instruction onset separately from that associated with generation of emotion at picture onset. Participants were instructed to follow the task instruction until cued to rate their success at doing so. Success ratings were provided on a four-point scale that was depicted visually for $5 \mathrm{~s}$ (1, no emotion; 2, not at all successful; 3 , somewhat successful; and 4, very successful).

\section{Data acquisition and reduction}

Functional and anatomical MRI. Whole-brain functional and anatomical images were acquired using a 3.0 tesla GE SIGNA MRI scanner (GE Medical Systems, Waukesha, WI) with LX software (version VH2), a transmit-receive quadrature birdcage coil, and Nvi $(40 \mathrm{mT} / \mathrm{m}$; slew rate, $150 \mathrm{mT} \cdot \mathrm{m}^{-1} \cdot \mathrm{ms}^{-1}$ ) gradients. Functional images were acquired using a T2*-weighted gradient-echo echo-planar imaging (EPI) pulse sequence with $4 \mathrm{~mm}$ sagittal slices [interslice gap, $1 \mathrm{~mm}$; echo time (TE), $30.0 \mathrm{~ms}$; repetition time (TR), $2000.0 \mathrm{~ms}$; flip angle, $60^{\circ}$; field of view (FOV), $240 \times 240 \mathrm{~mm} ; 64 \times 64$ data acquisition matrix]. The number of slices required to achieve whole-brain coverage ranged from 30 to 33 . With a TR of $2 \mathrm{~s}$ and a $17 \mathrm{~s}$ trial length, our effective temporal resolution was $1 \mathrm{~s}$. High-resolution T1-weighted spoiled gradient-echo three-dimensional anatomical images were collected in 124 contiguous $1.2 \mathrm{~mm}$ slices (TE, $8.0 \mathrm{~ms}$; TR, $21.0 \mathrm{~ms}$; flip angle, $30^{\circ}$; FOV, $240 \times 240 \mathrm{~mm} ; 256 \times 256$ data acquisition matrix), thus enabling localization of functional effects.

The functional MRI data were processed using the Analysis of Functional NeuroImages (AFNI version 2.40e) suite of image analysis routines. During off-line reconstruction, the first five whole-brain images were discarded from each of four scan runs to ensure steady-state magnetization. Because our interest centered on structures whose ventral locations are subject to susceptibility-related signal dropout, we limited our single-subject time series analyses to voxels meeting a signal threshold of at least $\sim 10 \%$ of the whole-brain maximum signal value for the first time point (after discarding the first five images). The raw EPI data were converted to AFNI format with correction for slice timing differences attributable to interleaved acquisition. Using six rigid body motion parameters, each time point was then registered to the one closest in time to collection of our anatomical images. We applied a high-pass filter $(0.02 \mathrm{~Hz})$ to remove slow drift in the signal over time and then concatenated the four scan runs into one single-subject dataset for each participant.

Single-subject general linear models (GLMs) using full deconvolution (B. D. Ward, Deconvolution Analysis of fMRI Time Series Data, http:// afni.nimh.nih.gov/afni) were performed to estimate the impulse response function for each of six conditions of interest (16 time points). In these omnibus analyses, stick-function predictors were used to indicate picture onset (negative, neutral), as well as regulation instruction onset (negative-increase, negative-decrease, negative-attend, and neutral-attend). Responses to picture onset versus regulation onset were statistically separable because of the 3-6 sitter in instruction onset. In addition, to account for variability in stimulus unpleasantness across conditions, we modeled normative ratings of unpleasantness gleaned from the IAPS dataset. Ratings were centered separately for the negative and neutral pictures so as to be orthogonal to the valence effects. [There was a small but reliable difference in stimulus unpleasantness such that, across sets, the negative pictures in the increase $(2.12 \pm 0.50)$ and attend conditions $(2.20 \pm 0.42)$ were more unpleasant than those in the decrease condition $(2.72 \pm 0.80)$. By modeling the normative ratings of unpleasantness (centered separately within the negative and neutral valence categories), we were able to statistically control for any small differences in blood oxygenation level-dependent (BOLD) signal that might be accounted for by unpleasantness rather than regulation instruction.] To account for motion-related change in BOLD signal, we also modeled the six motion parameters obtained during volume registration. Trials for which the pupil diameter data were missing for $>50 \%$ of the total trial length, likely indicating suboptimal viewing of the picture, were eliminated, as were trials that contained time points in which motion estimates exceeded 1.5 $\mathrm{mm}$.

A truncated area-under-the-curve (AUC) metric was calculated by summing estimates of percentage signal change $(100 \times \beta$ coefficient/ baseline) across an $8 \mathrm{~s}$ window after delivery of the regulation instruction (5-12 s, inclusive). This $8 \mathrm{~s}$ summary statistic maximizes chances of capturing the peak of the hemodynamic response even if the peak of the response varies temporally as a function of brain region and/or condition. Additionally, it eliminates noise associated with variation in the rise and fall of the response because those time points are excluded from the AUC summary statistic. Voxels were resampled to $2 \mathrm{~mm}^{3}$ and transformed into Talairach space. Estimates of AUC for percentage signal change were spatially blurred with a $5 \mathrm{~mm}$ fill-width half-maximal Gaussian filter.

To test for main effects of regulation condition, we conducted a voxelwise repeated-measures ANOVA in AFNI. We focused on negative trials only because no regulation instructions were provided during neutral trials. Statistically defined clusters of activation were identified using whole-brain Monte Carlo simulations (Alpha Sim program by AFNI) to achieve a corrected cluster threshold of $p<0.05$. Specifically, clusters reaching a contiguous volume of at least $296 \mathrm{~mm}^{3}$ at a voxelwise threshold of $p<0.001$ or $696 \mathrm{~mm}^{3}$ at a voxelwise threshold of $p<0.005$ were considered significant at $p<0.05$. Mean AUC estimates across all voxels in each cluster were extracted for each subject and submitted to repeatedmeasures GLM analyses and pairwise comparisons in SPSS version 12 (SPSS, Chicago, IL). To test a priori hypotheses about amygdala involvement in regulation, mean AUC estimates across all voxels in two (left and right) amygdala regions of interest (ROIs), defined using the Talairach Atlas provided with AFNI, were extracted for each subject and similarly submitted to follow-up GLM and pairwise analyses.

Salivary cortisol. Using the Salivette saliva collection device (Sarstedt, Newton, NC), a cotton roll housed within a clear plastic tube, participants collected six saliva samples per day for 7 consecutive days at home during the week immediately after their MRI scan session. On each day, the first sample was collected $0.5 \mathrm{~h}$ after awakening. Four additional samples were collected at random times within each of the following $3 \mathrm{~h}$ blocks of time: 9:00 A.M. to 12:00 P.M., 12:00 P.M. to 3:00 P.M., 3:00 P.M. to 6:00 P.M., and 6:00 P.M. to 9:00 P.M.. The final sample was collected at bedtime. Using this method, we ensured that we could adequately resolve the diurnal pattern while minimizing the chance that recurring daily events would systematically bias our estimates.

Samples were centrifuged for $10 \mathrm{~min}$ at $5000 \mathrm{rpm}$ to remove cellular and bacterial debris that are inherent in saliva samples. We then conducted a cortisol enzyme immunoassay (Salimetrics, State College, PA). Assay results were considered acceptable only if the coefficient of variation (CV) for the duplicate measurement of a sample was $\leq 20 \%$. The mean interassay $\mathrm{CV}$ was $7.4 \%$, and the mean intraassay $\mathrm{CV}$ was $3.8 \%$. The detection limit for this assay is $0.007 \mu \mathrm{g} / \mathrm{dl}$. Diurnal slope was calculated by regressing log-transformed cortisol values on time for each subject and day. Estimates of slope were then averaged across days and submitted to correlational and regression analyses to assess associations with the aggregate estimates of PFC and amygdala fMRI signal while regulating negative affect.

Pupil diameter. Horizontal pupil diameter was acquired continuously (sampling rate, $60 \mathrm{~Hz}$ ) during the scan session. The pupil dilation data were cleaned and processed using algorithms designed by Siegle, Granholm, and Steinhauer (2002, unpublished Matlab code) with Matlab software (MathWorks, Natick, MA) and adapted in our laboratory (L. L. Greischar, 2003, unpublished Matlab code). Blinks were identified and eliminated using local regression slopes and amplitude thresholds. Missing data points were then estimated using linear interpolation. A fivesample rolling average was calculated to smooth the signal, and slow, irrelevant drifts in the pupil diameter data over the course of the scan session were removed via linear detrending over blocks of 24 trials. Trials 
requiring $>50 \%$ interpolation during the picture presentation period as well as trials whose value fell greater than \pm 4 SD from the within-subjects mean were eliminated. Pupil diameter was aggregated into $0.5 \mathrm{~s}$ bins, and then means of the unstandardized pupil diameter residuals (removing variance associated with normative ratings of unpleasantness, the same strategy followed in our fMRI analysis) were computed for each epoch during the picture presentation period. These values were rangecorrected within subjects [(current pupil diameter - minimum pupil diameter $) \div$ (maximum pupil diameter - minimum pupil diameter)] and then aggregated within conditions across trials. Mean diameter for the $0.5 \mathrm{~s}$ picture period immediately before the regulation instruction was then subtracted from the mean pupil diameter during each of 10 0.5 -s picture periods after instruction up until picture offset within each condition. Proportional change in diameter was then computed for each of the 10 time points after the instruction [i.e., (post - pre)/pre].

We next computed across-subjects GLM analyses to assess the effect of regulation instructions on pupil diameter Although insufficient power precluded treating time as a factor in one omnibus test, we assessed temporal specificity of the increase, attend, and decrease instructions by separately evaluating activity averaged across time points within each of three post-instruction time bins [early $(0.5-2.0 \mathrm{~s}$ post-instruction average), middle (2.5-3.5 s post-instruction), and late $(4.0-5.0 \mathrm{~s}$ post-instruction)].

Behavioral data. Ratings of perceived success were averaged across trials within each regulation condition on a subject-by-subject basis. They were then submitted to a groupwise repeated-measures GLM.

\section{Results}

\section{Overview}

We first present the main effects of emotion regulation condition (increase, attend, decrease) on fMRI signal. These analyses address our first two predictions concerning PFC and amygdala involvement in voluntary emotion regulation. To address the third and fourth predictions concerning associations between the amygdala and MPFC, and between regions implicated in emotion regulation and salivary cortisol, we next present correlational and regression analyses. Finally, we present the main effects of emotion regulation condition on pupil diameter and ratings of perceived success.

\section{Amygdalar tracking of negative affect regulation}

We hypothesized that voluntary regulation of negative affect would linearly modulate amygdalar activation, with increasing yielding higher activation compared to attending, and attending, in turn, yielding higher activation compared to decreasing. Consistent with this pattern, a multivariate GLM testing the effect of condition (increase, attend, decrease) and hemisphere (left, right) on amygdalar response to negative pictures revealed a significant main effect of condition $\left(F_{(2,14)}=4.85 ; p=0.025\right)$ (Fig. $1)$ and a significant within-subjects condition linear contrast $\left(F_{(1,15)}=9.63 ; p=0.007\right)$. Mean AUC when increasing $(0.77 \pm$ 2.00) was higher than when both attending $(0.05 \pm 1.92 ; p=$ $0.017)$ and decreasing $(-0.16 \pm 2.27 ; p=0.007)$ in response to negative trials. Although mean AUC was higher for attend than decrease trials, this predicted effect was not reliable $(p=0.410)$. There was no effect of hemisphere $\left(F_{(1,15)}=0.01 ; p=0.919\right)$, nor was there a significant hemisphere $X$ condition interaction $\left(F_{(2,14)}=1.18 ; p=0.338\right)$.

\section{PFC involvement in regulation of negative affect}

As depicted in Figure 2, the voxelwise repeated-measures ANOVA testing the main effect of condition (increase, attend, decrease) on AUC in the BOLD response to negative pictures revealed frontal clusters (corrected cluster $p<0.05$ based on Monte Carlo simulations) in the left inferior frontal gyrus (IFG)

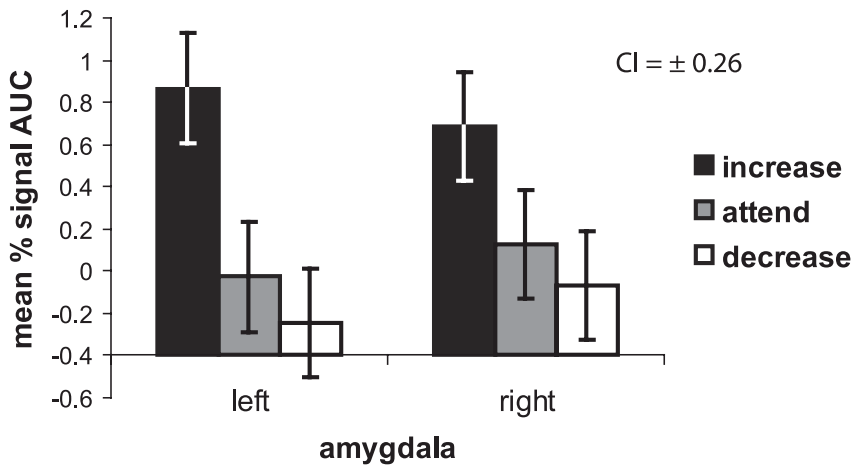

Figure 1. Depicted here is the response of the left and right amygdala. On the ordinate, we plot the AUC for percentage signal change estimates from 5 to $12 \mathrm{~s}$ after the instruction. Each regulation condition is represented on the abscissa. A main effect of condition indicates that increasing negative affect (black bar) produces larger signal change across the left and right amygdalae ( $n=16$ because of removal of one outlier in the right amygdala for the attend condition) compared to the other two conditions (attending, gray bars; decreasing, white bars). Error bars represent a $95 \%$ confidence interval $(\mathrm{Cl})$, which was computed on a within-subjects basis in accordance with Loftus and Masson (1994).

[Brodmann's area (BA) 44], which includes portions of Broca's area, a superior dorsal region of the left medial frontal gyrus (MFG) (BA 6), which includes portions of supplementary motor area, a more anterior dorsal region of the left MFG (BA 10), and the left superior frontal gyrus (SFG) (BA 9), part of the dorsolateral PFC. Mean AUC was computed across all voxels within each of these frontal regions and submitted to repeated-measures GLMs followed by pairwise comparisons. Mean AUC in the left IFG cluster when increasing $(1.70 \pm 0.96)$ was higher than when attending $(0.86 \pm 0.85 ; p<0.001)$ or decreasing $(0.95 \pm 0.84$; $p<0.001$ ) (Fig. 2a). The difference between decreasing and attending in the left IFG was not significant $(p=0.423)$. The same pattern (increase $>$ both attend and decrease; attend $=$ decrease) was also evident for the left SFG (increase, $0.78 \pm 0.79$; attend, $-0.01 \pm 0.84$; decrease, $-0.08 \pm 1.00$ ) (Fig. $2 b$ ). A linear pattern of effects, similar to that shown in the amygdala, was found in the anterior dorsal MFG (BA 10) (Fig. 2c). In this region, increasing $(0.86 \pm 1.52)$ generated higher mean AUC compared to attending $(0.07 \pm 1.32)$, and attending was higher in turn than decreasing $(-0.18 \pm 1.40)$. Finally, in the superior dorsal MFG/BA 6, both increasing $(2.91 \pm 1.66)$ and decreasing $(2.34 \pm 1.78)$ resulted in significantly higher activation relative to attending $(1.83 \pm 1.88)$, but increasing was still higher than decreasing in this cluster $(p<0.001)$ (Fig. $2 d)$.

\section{Regulation effects in other brain regions}

Table 1 presents a comprehensive list of findings that emerged in the voxelwise ANOVA, including the four regions described above that are also depicted in Figure 2 (noted in Table 1). The cluster with the greatest spatial extent, spanning multiple anatomical regions, was a left posterior medial cluster that included posterior cingulate gyrus, precuneus, and culmen. Also spanning multiple regions, the cluster with the second largest extent included left middle temporal and occipital gyri and the superior temporal gyrus (BA 19 and 39). Additional areas of activation were noted in the right and left fusiform gyrus, right and left thalamus, right and left caudate, and left hippocampus, parahippocampal gyrus, and insula. For each cluster, mean AUC estimates were calculated across all voxels identified in each of these clusters and submitted to a repeated-measures GLM, and significant results were followed up with pairwise comparisons. These 
a. Left Inferior Frontal Gyrus (BA 44)
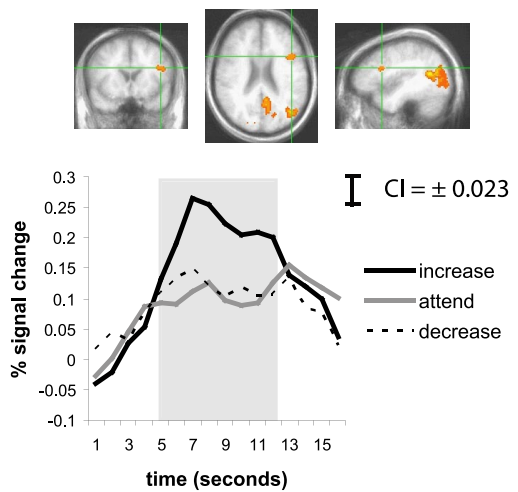

b. Left Superior Frontal Gyrus (BA 9)
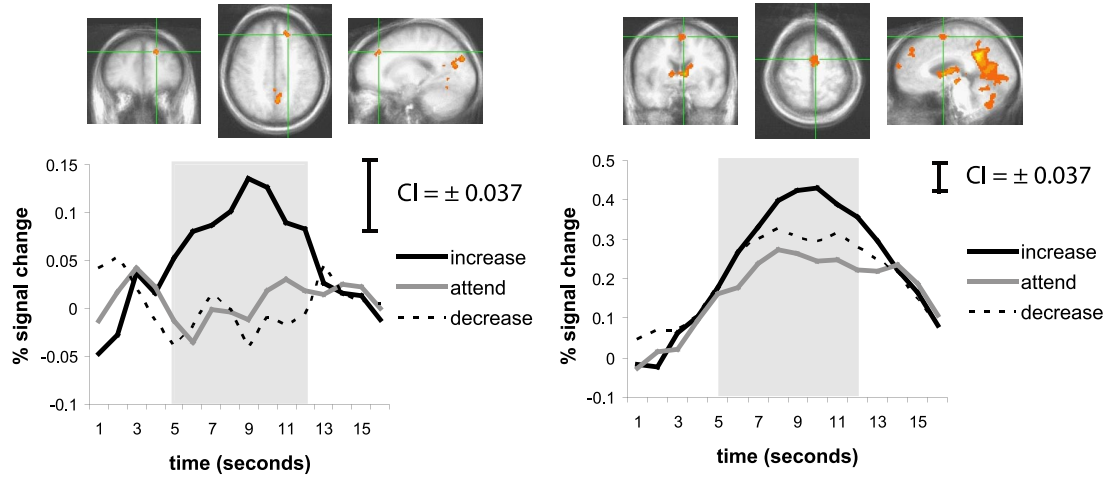

Figure 2. $\quad \boldsymbol{a}-\boldsymbol{d}$ depict the four prefrontal cortex regions (centered under the green crosshairs) that were responsive to the regulation instructions. $\boldsymbol{a}$, Left inferior frontal gyrus (BA 44). $\boldsymbol{b}$, Left superior frontal gyrus (BA 9). $\boldsymbol{c}$, Left anterior dorsal medial frontal gyrus (BA 10). $\boldsymbol{d}$, Left superior dorsal medial frontal gyrus (BA 6). For each region, there are three cardinal views of the statistically defined cluster of interest (coronal, axial, and sagittal) as well as the estimated impulse response function for the increase (black line), attend (gray line), and decrease (dotted line) instructions in response to negative pictures. Responses were quantified as the AUC of percentage signal change estimates from 5 to $12 \mathrm{~s}$ after the instruction, a period indicated by the gray shading in each graph. Confidence intervals (Cl;95\%), computed on a within-subjects basis in accordance with Loftus and Masson (1994) and scaled to represent each time point, are depicted at the top right of each line graph. The Talairach coordinates of the maximum $F$ statistic for each region are presented in Table 1.

results are organized in Table 1 according to the pattern of differences between averaged responses in each cluster for the three regulation conditions (increase, attend, decrease). With the exception of the two dorsal MFG clusters (anterior and superior) described previously (Table 1 , bottom), the effect of regulation condition was the same: increasing resulted in higher mean AUC compared to both attending and decreasing, which were not significantly different from one another. There were no main effects of regulation condition evident in VMPFC.

\section{Inverse associations between amygdala and VMPFC}

To determine whether MPFC regions show reciprocal coupling with amygdala activation, particularly when decreasing negative affect as predicted, we first calculated the MR signal difference in the amygdala during instructions to regulate relative to the attend control condition. Negative scores in the amygdala for the decrease-attend contrast (i.e., decrease $<$ attend) indicate more effective attenuation of negative affect. Positive scores in the amygdala for the increase-attend contrast (i.e., increase $>$ attend) indicate more effective amplification of negative affect. We then used this amygdala signal difference score as a groupwise predictor of the same signal difference in the remainder of the brain volume on a voxelwise basis.
Decrease-attend

As shown in Figure $3 a$, participants who most successfully reduced MR signal in the left amygdala showed the highest activation in both right and left VMPFC (BA 11 and 32) (maximum, $t_{(16)}=-5.28$ at Talairach coordinates $x=5, y=37, z=-12$; and maximum, $t_{(16)}=-4.79$ at $x=-23$, $y=43, z=-10$, respectively). Although a similar pattern was observed using the right amygdala as a predictor, it did not survive cluster thresholding.

\section{Increase-attend}

No significant associations were evident in parallel analyses using the increase-attend signal difference in the amygdala (left or right) as a predictor of the voxelwise increase-attend signal difference.

\section{Specificity}

The associations reported above between the left and right VMPFC and the amygdala appear to be specific to the decreaseattend contrast as opposed to representing a more general effect of engaging in regulatory processes. However, to test this more rigorously, we next performed two hierarchical multiple regression analyses. In the first analysis, we entered increaseattend activation in the left amygdala on the first step and increase-attend activation in the left VMPFC on the second step. (Increase-attend activation for VMPFC was extracted from the clusters identified in the voxelwise decrease-attend regression analysis described in the preceding paragraph.) On the third and final step, we entered decrease-attend activation in the left VMPFC. In the second analysis, we observed the same structure, this time entering right rather than left VMPFC on the second and third steps. Supporting a unique decrease-attend effect, results indicate that, even after removing variance explained by increase-attend activation in the left VMPFC and left amygdala, decrease-attend activation in the left VMPFC still explains $44 \%$ of the variance in decrease-attend activation in the left amygdala $\left(\Delta F_{(1,13)}=20.71\right.$; $p=0.001)$. Similarly, the right VMPFC still explains $26 \%$ of the variance in decrease-attend activation in the left amygdala $\left(\Delta F_{(1,13)}=11.15 ; p=0.005\right)$. In both analyses, increase-attend activation in neither the left nor the right VMPFC nor in the left amygdala explains unique variance in decrease-attend activation in the left amygdala (all four $p$ values $>0.40$ ).

\section{Does VMPFC mediate the association between dorsal PFC and the amygdala?}

The inverse association between the amygdala and VMPFC suggested that VMPFC might be exerting a top-down inhibitory effect on the amygdala. In our next set of analyses, we tested whether VMPFC might also be serving the role of intermediary between the amygdala and other regions of PFC that were implicated in emotion regulation (even those for which their involvement was apparently limited to the increase condition). Interestingly, higher activation in this anterior dorsal MFG (BA 10) 
Table 1. Statistically significant clusters emerging from the voxelwise repeated-measures ANOVA testing the main effect of regulation condition (increase, attend, decrease) on BOLD response

\begin{tabular}{|c|c|c|c|c|c|}
\hline \multirow[b]{2}{*}{ Brain region (Brodmann area) } & \multirow{2}{*}{$\begin{array}{l}\text { Cluster size } \\
\left(\mathrm{mm}^{3}\right)\end{array}$} & \multicolumn{3}{|c|}{ Talairach coordinates at maximum $F$} & \multirow[b]{2}{*}{ Fvalue of maximum } \\
\hline & & $x$ & $y$ & $z$ & \\
\hline \multicolumn{6}{|l|}{ Increase $>$ attend and decrease } \\
\hline L. cingulate gyrus, precuneus, culmen (31) & 8416 & -5 & -47 & 28 & 31.11 \\
\hline \multicolumn{6}{|l|}{ L. middle temporal gyrus, middle occipital gyrus, } \\
\hline superior temporal gyrus $(19,39)$ & 6632 & -41 & -53 & 14 & 30.68 \\
\hline R. fusiform gyrus, middle occipital gyrus, declive (37) & 3600 & 41 & -61 & -12 & 24.19 \\
\hline L. medial and superior frontal gyrus $(8,9)$ & 2480 & -15 & 39 & 36 & 14.98 \\
\hline L. thalamus, caudate & 1904 & -3 & -9 & 10 & 20.06 \\
\hline L. fusiform gyrus (37) & 1488 & -35 & -51 & -12 & 20.93 \\
\hline L. cuneus, precuneus (18) & 1208 & -19 & -83 & 26 & 16.92 \\
\hline L. culmen, declive & 1048 & -9 & -53 & -16 & 17.91 \\
\hline R. cuneus $(17,18)$ & 960 & 7 & -81 & 16 & 13.84 \\
\hline R. uvula & 896 & 11 & -61 & -26 & 19.94 \\
\hline R. thalamus, caudate & 840 & 11 & 1 & 4 & 16.01 \\
\hline R. cuneus, precuneus, middle occipital gyrus $(18,19)$ & 768 & 29 & -75 & 18 & 13.19 \\
\hline R. middle temporal gyrus (37) & 696 & 45 & -57 & 6 & 18.96 \\
\hline L. hippocampus, parahippocampal gyrus, insula & 624 & -37 & -13 & -8 & 19.37 \\
\hline L. inferior frontal gyrus (44) (Fig. 2a) & 536 & -45 & 11 & 22 & 18.39 \\
\hline L. superior frontal gyrus (9) (Fig. 2b) & 536 & -15 & 39 & 36 & 14.98 \\
\hline R. culmen, fusiform gyrus & 424 & 37 & -45 & -18 & 15.45 \\
\hline \multicolumn{6}{|l|}{ Increase $>$ attend $>$ decrease } \\
\hline L. medial frontal gyrus (10) (Fig. 2c) & 1040 & -3 & 63 & 18 & 13.06 \\
\hline \multicolumn{6}{|l|}{ Increase and decrease $>$ attend } \\
\hline L. medial frontal gyrus (6) (Fig. 2d) & 824 & -3 & -7 & 58 & 14.41 \\
\hline
\end{tabular}

Pairwise comparisons were conducted on mean AUC across all voxels in each cluster to determine the pattern of differences between the three conditions. L, Left; $R$, right.

region was associated with higher activation in the left and right VMPFC when decreasing relative to attending $\left(r_{(16)}=0.70, p=\right.$ 0.002 ; and $r_{(16)}=0.61, p=0.009$, respectively). In addition, subjects demonstrating higher decrease-attend activation in anterior dorsal MFG (BA 10) showed lower decrease-attend activation in the left amygdala $\left(r_{(15)}=-0.54 ; p=0.03\right)$. Decreaseattend activations in the left superior dorsal MFG (BA 6), left SFG, and left IFG were uncorrelated with one another, nor were they correlated with left amygdalar activation for decrease-attend ( $p$ values $>0.10$ ). These results suggest that the only candidate region possibly acting on VMPFC was the anterior dorsal MFG (BA 10) because it is the only one to be associated with both VMPFC and the amygdala. [For the sake of completeness, acrosssubject correlations between the statistically defined frontal regions of interest identified in our tests for regulation main effects (anterior and superior dorsal MFG, SFG, and IFG) and between those regions and the a priori amygdala ROI are presented in Table 2.]

We next conducted the Sobel test of mediation as described and implemented by Preacher and Hayes (2004). In brief, this test provides a means of calculating and testing the significance of the indirect effect of a predictor variable (BA 10) on a criterion variable (amygdala) by way of a hypothesized mediator (VMPFC). This test supported a mediating role for the VMPFC between the anterior dorsal MFG (BA 10) and the amygdala $(Z=-2.86, p=$ 0.0042 for left VMPFC; and $Z=-2.33, p=0.012$ for right VMPFC). Moreover, as a test of specificity, reversing the order (i.e., treating VMPFC as the predictor and BA 10 as the mediator) suggested that BA 10 does not account for the association between VMPFC and the amygdala $(Z=0.48, p=0.63$ for left VMPFC; and $Z=-0.30, p=0.76$ for right VMPFC). As suggested by the absence of relevant correlations, the VMPFC did not exhibit a mediating relationship with any of the other frontal regions identified earlier [i.e., SFG, IFG, or superior dorsal MFG (BA 6)] and the amygdala.

\section{Patterns of cortisol secretion}

Salivary cortisol, expressed in log-transformed micrograms per deciliters exhibited the expected pattern of declining values over the course of the day, as indicated by negative values of diurnal slope averaged across study days in all participants $(-0.17 \pm$ $0.05)$. Levels of cortisol secretion were significantly higher in the first morning sample, taken $30 \mathrm{~min}$ after awakening $(-0.35 \pm$ 0.17 ) compared with the last sample of the day, taken just before bedtime $\left(-1.25 \pm 0.20 ; t_{(15)}=12.28 ; p<0.001\right)$. These morning and bedtime samples were not significantly correlated in this sample $\left(r_{(15)}=-0.23 ; p=0.40\right)$, but together they explained $94 \%$ of the variance in diurnal slope $\left(F_{(2,13)}=107.36 ; p<0.001\right)$, with higher values in the morning being associated with steeper (more negative) slopes $(\beta=-0.49 ; p<0.001)$ and higher values in the evening being associated with flatter (less negative) slopes $(\beta=0.73 ; p<0.001)$.

\section{Cortisol secretion and its association with emotion regulatory brain responses}

We next examined groupwise correlations between brain responses during emotion regulation and the diurnal slope of salivary cortisol. Regions of interest for these analyses were the left and right amygdala ROIs described previously, and the PFC clusters identified either in the voxelwise regression analysis treating left amygdala as a predictor (left and right VMPFC) and in the voxelwise test of regulation main effects (SFG, IFG, anterior dorsal MFG, and superior dorsal MFG).

\section{Decrease-attend}

As depicted in Figure $3 b$, we found that individuals who showed more activation in the left and right VMPFC when decreasing 


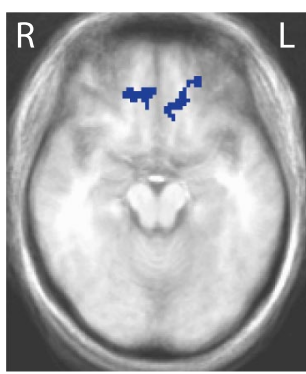

b. cortisol slope with lef VMPFC

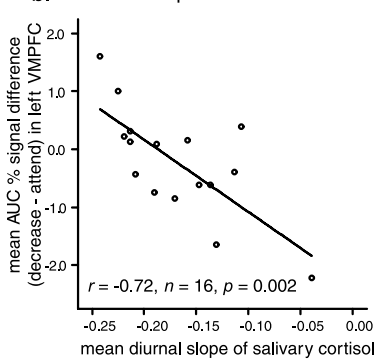

left amygdala with left VMPFC

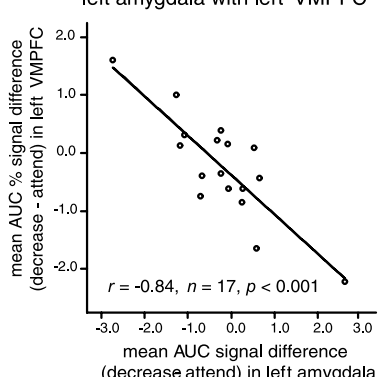

cortisol slope with right VMPFC

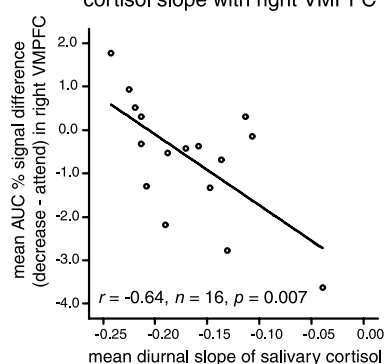

left amygdala with rightVMPFC
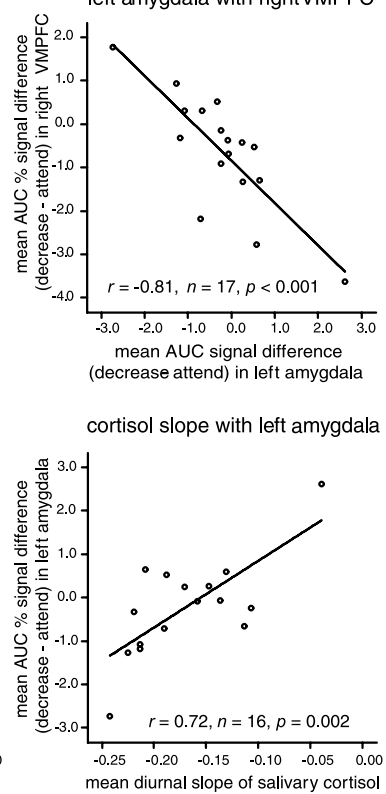

Figure 3. $\boldsymbol{a}$, Two regions in left $(\mathrm{L})$ and right (R) VMPFC (maximum, $t_{(16)}=-4.79$ at Talairach coordinates $x=-23, y=43$, $z=-10$; and maximum, $t_{(16)}=-5.28$ at $x=5, y=37, z=-12$, respectively) demonstrate an inverse across-subjects correlation with the left amygdala. For illustrative purposes, we extracted mean signal across all voxels for the decrease and attend conditions separately for the two VMPFC regions and also for the left amygdala. We then computed zero-order correlations between the amygdala and VMPFC across subjects and depicted those associations in the accompanying scatter plots. Subjects with lower activation in the amygdala (top middle and right) (abscissa) exhibit higher activation in the right and left VMPFC (ordinate). Represented on all axes is the difference in AUC for percentage signal change between the decrease and attend conditions (decrease-attend). $\boldsymbol{b}$, We next computed zero-order, across-subjects correlations between diurnal cortisol slope (abscissa) and the difference in AUC for percentage signal change between the decrease and attend conditions (decrease-attend) across all voxels in the left and right VMPFC (bottom left and middle) and the amygdala (bottom right) (ordinate) clusters. Subjects with higher activation in VMPFC and lower activation in the amygdala exhibit the steepest declines in salivary cortisol over the course of the day.

Table 2. Across-subjects correlations between regions for regulation condition contrasts (decrease-attend and increase-attend) in BOLD signal

\begin{tabular}{|c|c|c|c|c|c|}
\hline Decrease-attend contrast & 1 & 2 & 3 & 4 & 5 \\
\hline \multicolumn{6}{|l|}{ Left SFG (BA 9) } \\
\hline Left IFG (BA 44) & 0.28 & & & & \\
\hline Left superior MFG (BA 6) & 0.36 & -0.24 & & & \\
\hline Left anterior MFG (BA 10) & -0.08 & -0.11 & -0.29 & & \\
\hline Left amygdala & 0.27 & 0.17 & -0.05 & $-0.54^{*}$ & \\
\hline Right amygdala & -0.002 & 0.08 & -0.04 & $-0.45^{+}$ & $0.56^{*}$ \\
\hline $\begin{array}{l}\text { Increase-attend contrast } \\
\text { Left SFG (BA 9) }\end{array}$ & 1 & 2 & 3 & 4 & 5 \\
\hline Left IFG (BA 44) & -0.09 & & & & \\
\hline Left superior MFG (BA 6) & $0.62^{* *}$ & 0.36 & & & \\
\hline Left anterior MFG (BA 10) & $0.63^{* *}$ & -0.23 & 0.14 & & \\
\hline Left amygdala & 0.23 & 0.28 & 0.27 & $0.46^{+}$ & \\
\hline Right amygdala & 0.36 & 0.36 & 0.29 & $0.58^{*}$ & $0.73^{* *}$ \\
\hline
\end{tabular}

Correlations were computed for the decrease-attend and increase-attend contrasts for the four statistically defined frontal ROIs and the two Talairach Atlas-defined amygdala ROIs. $n=17$ for all but correlations with right amygdala, for which $n=16$ because of removal of one extreme outlier. * Correlation is significant at the 0.05 level, two-tailed. ${ }^{* *}$ Correlation is significant at the 0.01 level, two-tailed. ${ }^{+}$Correlation shows a trend at the 0.10 level, two-tailed.

relative to attending $(r=-0.72, n=16, p=0.002$ (bottom left); and $r=-0.64, n=16, p=0.007$ (bottom middle), respectively) and smaller activation in the left amygdala when decreasing relative to attending $(r=0.72 ; n=16 ; p=0.002$ (bottom right)] exhibited sharper declines in cortisol over the course of the day. Although activation in the right amygdala was also positively associated with cortisol slope, this correlation was not significant. Of the four frontal clusters identified in the test of regulation main effects (SFG, IFG, and anterior and superior dorsal MFG), only the anterior dorsal MFG (BA 10) exhibited any significant associations with cortisol. Interestingly, the pattern of associations mirrored those uncovered between VMPFC and cortisol. Specifically, subjects with higher activation in anterior dorsal MFG when decreasing relative to attending exhibited steeper declines in cortisol over the course of the day $(r=-0.57 ; n=16 ; p=0.02)$.

\section{Increase-attend}

Two significant correlations were detected between increase-attend brain responses for the same regions tested above and salivary cortisol slope. Lower activation in the left amygdala when increasing relative to attending was associated with steeper declines in cortisol over the course of the day $(r=0.58 ; n=$ $16 ; p=0.017)$. Conversely, lower increase-attend activation in the right VMPFC was associated with flatter declines in cortisol $(r=-0.53 ; n=16 ; p=0.037)$. These findings are in the same direction as those reported above for the decreaseattend contrast.

\section{Specificity}

Because the direction of associations between cortisol slope and brain responses in the left amygdala and right VMPFC while increasing (relative to the attend control condition) is the same as those between cortisol slope and decreasing negative affect, it is possible that processes in these regions related to regulatory efforts in general are the key to exhibiting adaptive patterns of diurnal cortisol secretion. To address this question, we next conducted two hierarchical multiple regression analyses to determine whether decrease-attend activation in these two regions predicts unique variance in cortisol slope after removing variance associated with increaseattend activation. In the first analysis, we entered increase-attend and decrease-attend activation in the left amygdala as predictors of cortisol slope. Results indicate that decrease-attend activation in the left amygdala uniquely explains $27 \%$ of the variance in cortisol slope $\left(\Delta F_{(1,13)}=8.74 ; p=0.011\right)$, even after removing variance explained by increase-attend activation in the left amygdala, which itself is not significant $(\beta=0.33$; $p=$ 0.12 ). In the second analysis, we entered increase-attend and decrease-attend activation in the right VMPFC as predictors of cortisol slope. Results indicate that decrease-attend activation in the right VMPFC uniquely explains $20 \%$ of the variance in cortisol slope $\left(\Delta F_{(1,13)}=4.94 ; p=0.045\right)$, even after removing variance explained by increase-attend activation in the right VMPFC, which itself is not significant $(\beta=-0.28 ; p=0.24)$. 
Unique contributions of morning versus evening cortisol values Simultaneous regression analyses, in which we treated the first morning and bedtime cortisol samples as predictors of activation in VMPFC and the amygdala, suggested that bedtime cortisol levels may be more important than morning cortisol levels in associations between these regions and diurnal cortisol slope. Specifically, bedtime $(\beta=-0.58 ; p=0.013)$ but not morning $(\beta=0.28 ; p=0.20)$ cortisol levels explained unique variance in left VMPFC when decreasing relative to attending to negative affect. A similar pattern of associations was evident for right VMPFC for bedtime $(\beta=-0.45 ; p=0.065)$ and for morning $(\beta=0.33 ; p=0.17)$. Finally, bedtime $(\beta=0.60 ; p=0.012)$ but not morning $(\beta=-0.22 ; p=0.30)$ cortisol levels explained unique variance in the left amygdala when decreasing relative to attending. The same direction of associations as noted above for VMPFC was discovered for anterior dorsal MFG, although neither morning $(\beta=0.32 ; p=0.21)$ nor bedtime $(\beta=-0.38$; $p=$ 0.14 ) cortisol levels reached significance in this region. We did not conduct parallel analyses for the increase-attend contrast because no unique brain-cortisol associations were uncovered above.

\section{Signal quality}

The ventral nature of the VMPFC and amygdala regions described above increases the risk of susceptibility-related signal drop-out relative to their dorsal counterparts. Moreover, our sample is older; thus, although the age range is restricted to just a few years in the present sample, one might expect more morphometric variation than would be evident in a younger sample. Signal drop-out and morphometric variation would both be evident in variation across subjects in baseline BOLD signal, in which case it was possible that baseline signal might explain the inverse between-subjects associations between the VMPFC and left amygdalar regions for the decrease-attend signal difference. We adopted a number of strategies to rule out this possibility. For one, as noted in Materials and Methods, we limited our singlesubject time series analyses to voxels meeting a signal threshold of at least $\sim 10 \%$ of the whole-brain maximum signal value for the first time point (after discarding the first five images to ensure steady-state magnetization). Second, on a groupwise basis, we extracted estimates of baseline signal for the right and left VMPFC and for the left amygdala and computed zero-order correlations between baseline signal and decrease-attend signal in each region. No significant correlations emerged ( $p$ values $>0.30$ ). We also used the baseline values as control variables in hierarchical regression analyses. Activation in left and right VMPFC clusters for the decrease-attend difference continued to predict unique variance in left amygdala signal for the decreaseattend comparison even when separately controlling for variation in baseline signal in these regions $\left(\beta=-0.86, \Delta R^{2}=0.72\right.$, $\Delta F_{(1,13)}=35.58, p<0.001$ for right VMPFC; and $\beta=-0.90$, $\Delta R^{2}=0.76, \Delta F_{(1,13)}=44.82, p<0.001$ for left VMPFC). Variation in baseline signal as a function of susceptibility artifact and/or volumetric variation in this aged sample does not therefore explain the inverse association between VMPFC and amygdala reported above. However, this does not rule out the possibility that morphometric variation might still be at work because morphometric changes may be manifest in task-related functional activations and not in the baseline state.

\section{Medication usage}

All participants reported taking at least one form of medication within 2 weeks of the scan session, not unsurprising given their
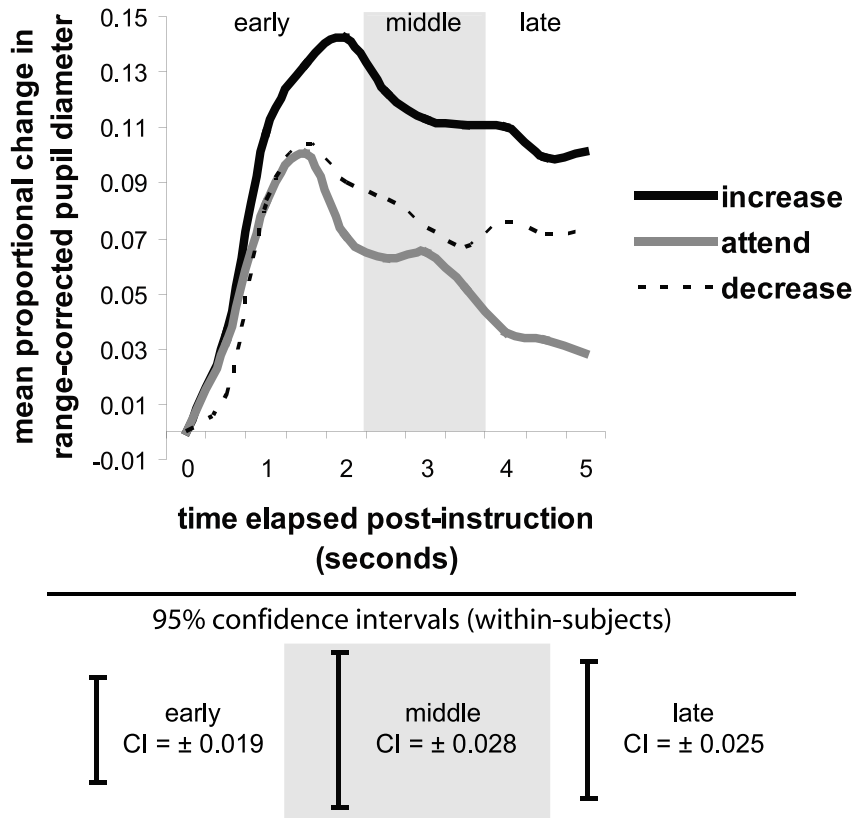

Figure 4. Depicted here is proportional change in pupil diameter, reported separately for the increase (black line), attend (gray line), and decrease (dotted line) instructions in response to negative pictures. The shaded panel delineates the middle regulation period $(2.5-3.5 \mathrm{~s})$, with early $(0.5-2 \mathrm{~s})$ and late $(4-5 \mathrm{~s})$ periods appearing as unshaded before and after, respectively. Confidence intervals (Cl; $95 \%)$, computed separately for the early, middle, and late regulation periods on a within-subjects basis in accordance with Loftus and Masson (1994), are depicted beneath the line graph.

advanced age. Medications reported include the following: antiarthritis $(n=5)$, anti-cholesterols $(n=6)$, antidepressants $(n=$ $2)$, anti-hypertensives $(n=5)$, thyroid hormones $(n=7)$, estrogen hormones $(n=6)$, insulin $(n=5)$, and/or antibiotics $(n=$ 2 ). This raises the possibility that medication usage may account for some of the observed covariation between VMPFC and the amygdala and/or between these regions and cortisol. However, inspection of the zero-order scatter plots suggested that these subjects did not carry the effects reported. In addition, excluding the individuals reporting use of these medications in the past 2 weeks does not affect the pattern of results. In all cases, the direction of the associations is preserved, and, in most cases, the correlations remain significant or nearly so despite the loss of statistical power.

\section{Regulation-induced changes in pupil diameter}

Figure 4 presents proportional change in pupil diameter over time after delivery of increase, attend, and decrease instructions on negative picture trials. We predicted that increasing and decreasing negative affect would result in greater proportional increases in pupil diameter compared to attending. Suggesting sustained processing during the two active forms of regulation, both increasing $(0.10 \pm 0.072)$ and decreasing $(0.07 \pm 0.047)$, resulted in larger pupil diameter compared to attending $(0.03 \pm 0.053$; $p=0.002$ and 0.043 , respectively), during the late regulation period $\left(F_{(2,12)}=7.31 ; p=0.008\right)$. Moreover, the difference in pupil diameter between increasing and decreasing during the late regulation period only bordered on significant $(p=0.085)$. In the early $\left(F_{(2,12)}=6.46 ; p=0.012\right)$ and middle $\left(F_{(2,12)}=4.91\right.$; $p=0.028$ ) (Fig. 4 , gray shading) regulation periods, increasing negative affect $(0.10 \pm 0.049$ and $0.12 \pm 0.064$, respectively) resulted in larger pupil diameter compared to both attending $(0.07 \pm 0.043$ and $0.06 \pm 0.054)$ and decreasing $(0.07 \pm 0.037$ 
Table 3. Across-subjects correlations between BOLD response and proportional change in pupil diameter for the frontal and amygdalar clusters for the regulation condition contrasts (decrease-attend and increase-attend)

\begin{tabular}{|c|c|c|c|c|c|c|}
\hline \multirow[b]{2}{*}{ Regulation period } & \multicolumn{6}{|l|}{ Region } \\
\hline & Left SFG (BA 9) & Left IFG (BA 44) & Left MFG (BA 6) & Left MFG (BA 10) & Left amygdala & Right amygdala \\
\hline \multicolumn{7}{|c|}{ Decrease-attend contrast } \\
\hline Early & $0.76^{* *}$ & $0.59 *$ & 0.09 & -0.27 & -0.36 & 0.44 \\
\hline Middle & $0.62^{*}$ & 0.41 & 0.06 & -0.03 & -0.22 & 0.14 \\
\hline Late & $0.63^{*}$ & $0.63^{*}$ & 0.10 & -0.03 & $-0.54^{+}$ & 0.28 \\
\hline \multicolumn{7}{|c|}{ Increase-attend contrast } \\
\hline Early & 0.17 & -0.04 & -0.20 & 0.37 & -0.03 & 0.08 \\
\hline Middle & 0.15 & 0.29 & -0.09 & 0.28 & 0.32 & 0.09 \\
\hline Late & -0.27 & 0.20 & -0.32 & -0.27 & 0.06 & -0.06 \\
\hline
\end{tabular}

Correlations were computed for the decrease-attend and increase-attend contrasts for both data types. Correlations were computed between pupil diameter and the four statistically defined frontal R0Is and the two Talairach Atlas-defined amygdala ROIs. For decrease-attend, $n=12$. Data for one subject were excluded for increase-attend because of extreme outlier status on several variables; thus, $n=11$. Early, $0.5-2.0$ s after instruction; Middle, $2.5-3.5 \mathrm{~s}$ after instruction; Late, $4.0-5.0$ s after instruction. ${ }^{*}$ Correlation is significant at the 0.05 level, two-tailed. ${ }^{*}$ Correlation is significant at the 0.01 level, two-tailed. ${ }^{+}$Correlation shows a trend at the 0.10 level, two-tailed.

and $0.08 \pm 0.047 ; p$ values $<0.05)$. There was no difference in pupil diameter for decreasing versus attending during the early and middle time periods ( $p$ values $>0.40$ ).

\section{Associations between brain activation and pupil diameter}

The fMRI analyses identified several regions of PFC and the amygdala that are more active when participants increase or decrease negative affect. Moreover, having just shown that increasing and decreasing negative affect produce significant changes in pupil diameter, we next examined an exploratory question: to what extent is the role of these regions in affect regulation associated with the degree of effort required by the task? We turned to our pupil diameter data to address this question. First, to index individual differences in the extent to which participants expended effort in the two active regulation conditions controlling for attention to negative information, we computed two pupil diameter difference scores: increase-attend and decrease-attend. A positive number indicates greater effort for increase or decrease relative to the control condition. We then calculated correlations between each of these difference metrics and its isomorphic contrast in brain activation for the frontal and amygdalar clusters identified above, regions of a priori interest given their role in regulation of negative affect in this study. Table 3 summarizes these associations. Notably, subjects showing higher activation in the left IFG and left SFG clusters for the decrease-attend contrast also showed higher decrease-attend pupil diameter difference scores in early, middle, and late regulation periods. Conversely, subjects showing higher activation in the left amygdala for the decrease-attend contrast showed lower scores on the decreaseattend pupil diameter difference in the late regulation period, an effect that nearly surpassed the significance threshold. There were no significant correlations between increase-attend activation in the frontal and amygdalar clusters identified in our main effect analyses and the increase-attend pupil diameter difference scores.

\section{Behavioral data}

A multivariate GLM testing the effect of condition (increase, attend, or decrease in response to negative stimuli) on ratings of task success, in which ratings ranged from 1 to 4 , revealed no significant effect $(p>0.20$; increase, $2.91 \pm 0.61$; attend, $2.76 \pm$ 0.73 ; decrease, $2.73 \pm 0.57)$.

\section{Discussion}

From this work, we draw three conclusions about the nature of the neural and endocrine correlates of emotion regulation, which we operationalized as the use of cognitive reappraisal to increase and decrease felt emotion in response to unpleasant visual stimuli. First, changes in fMRI signal in the amygdala and regions of $\mathrm{PFC}$ when attempting to reduce the experience of negative affect, specifically in an anterior region of BA 10 within the medial frontal gyrus and bilateral VMPFC, are lawfully related to diurnal cortisol secretion in the home environment. Second, experimenter-cued (but participant-generated) reframing of negative visual stimuli produces effects in lateral/dorsal PFC and the amygdala in older adults that are similar to, but less robust than, those reported in some previous studies with younger adults. Third, inverse functional coupling between VMPFC and amygdala regions during negative affect reduction is consistent with the conclusion that VMPFC exerts a top-down inhibitory influence on the activity of the amygdala. Importantly, BA 10 exerts an indirect effect on the amygdala that is mediated by bilateral regions of VMPFC.

Individuals who were good at diminishing negative affect, as evidenced by higher VMPFC and lower amygdala activation, exhibited a more normative (i.e., steeper) decline in salivary cortisol over the course of the day, with bedtime cortisol levels being more important than morning levels in this regard. These findings likely do not reflect regulatory skill or general cognitive task performance because accounting for variance associated with intensifying negative affect, itself not a significant predictor, did not obliterate these effects. Animal studies suggest that chronic stress is associated with elevated glucocorticoid secretion in the evening but not in the morning (Marti et al., 1993; Janssens et al., 1995). Additionally, dysregulation of cortisol secretion is evident in psychopathological conditions such as posttraumatic stress disorder and major depression (Burke et al., 2005; Yehuda et al., 2006). As reviewed by Sullivan and Gratton (1999), MPFC is rich in glucocorticoid receptors and has been implicated in negative feedback inhibition of the HPA axis. Disrupted negative feedback inhibition may produce chronic dysregulation of cortisol secretion (Diorio et al., 1993; McEwen, 1999; Sapolsky, 2003), which itself may contribute to disease states (Sephton et al., 2000). This pattern of findings is consistent with the idea that (1) a circuit involving the VMPFC and the amygdala underlies effective emotion regulation ability, and (2) this circuit mediates HPA disturbances associated with chronic stress and psychopathology. In subsequent waves of data collection within the Wisconsin Longitudinal Study, participants in the current study will complete measures of health and well-being that will enable us to use the present brain data to test whether activity in these circuits also predicts longer-term physical and mental health outcomes.

In previous studies, deliberate attenuation of negative affect 
yielded activation in dorsolateral, ventral lateral, and dorsomedial (including dorsal anterior cingulate cortex) regions of PFC, a finding that has been interpreted to reflect the execution of cognitive control for regulatory purposes (Ochsner et al., 2004; Phan et al., 2005). Interestingly, our analyses of condition main effects yielded only one region in which decreasing negative affect yielded stronger activation than unregulated attention (i.e., supplementary motor area), and even here higher activation was observed when increasing compared with decreasing negative affect. The most striking difference between this study and those reported previously is the advanced age of our sample. Advanced age is associated with changes in brain structure and/or function as well as with differences in cognitive and emotional processing. Aging may therefore be an important factor in understanding the paucity of frontal main effects with decreasing negative affect in this report. The inconsistency with previous work might also reflect the mixed gender nature of our sample and differences in design and methodology. Space considerations preclude an extended discussion of these issues here, but interested readers may refer to the supplemental material (available at www.jneurosci.org).

Whereas we find few PFC effects for the decrease condition, correlational analyses indicate that subjects who allocated more cognitive resources to decreasing than attending to negative affect, as indexed by larger pupil diameters, exhibited more activation in the SFG and IFG and less activation in the amygdala when decreasing than attending. Thus, whereas the involvement of these regions in negative affect reduction was not supported in tests of main effects, lawful associations with cognitive processing demands support the effectiveness of the manipulation. Similarly, lawful associations were also identified between the amygdala and VMPFC when participants deliberately diminished their affective response. Relative to their responses when passively experiencing negative affect, individuals who effectively reduced amygdalar responses when decreasing negative affect exhibited activation in bilateral VMPFC, an effect that was not evident anywhere else in PFC nor when increasing negative affect. Ventromedial PFC has been implicated in human and animal studies of conditioned fear extinction (Milad and Quirk, 2002; Phelps et al., 2004). In addition, Kim et al. (2003) demonstrated that VMPFC and amygdala responses are inversely correlated during processing of surprised facial expressions. Importantly, stimulation of the rodent analog of VMPFC reduces responses in the central nucleus of the amygdala (Quirk et al., 2003).

Based on the literature reviewed above, it should not be surprising that the VMPFC was implicated in the ability of subjects to transform their experience of negative stimuli to be less negative. Notably, the VMPFC regulatory effect is not observed as a mean difference; rather, it was only observed in analyses that harnessed variation across individuals. Whereas significant mean differences between conditions are less likely in the face of such variability, we discovered that those who are good at attenuating negative affect (i.e., they display less activation in the amygdala; decrease $<$ attend) are the same ones exhibiting greater bilateral activation in VMPFC (decrease $>$ attend). Because we control for emotional responses that occur spontaneously by subtracting out responses in the attend condition, this inverse association reflects an emotion regulatory as opposed to reactivity effect in this particular experimental context.

Interestingly, VMPFC appeared to serve a bridging function between the anterior dorsal MFG (BA 10) and the amygdala. Recent theories of BA 10 function suggest that this region is involved in coordination of information processing and informa- tion transfer when multiple processes are engaged in the service of a behavioral goal (Ramnani and Owen, 2004) and in coordinating attention between external stimuli and internal thoughts (Burgess et al., 2005). These functions match those required to regulate negative affect in our emotion regulation task, a task that likely involves multiple streams of information processing that would require such coordination, including maintaining representations of the behavioral goal to decrease negative affect, monitoring and modifying spontaneous appraisals arising in response to the images, and evaluating behavioral success. Although promising, the triangulation of associations between BA 10 and the amygdala, with VMPFC playing a mediating role, was not predicted on an a priori basis; thus, cautious interpretation is warranted. Moreover, although the pattern described above is consistent with a top-down process, the correlational nature of these associations and the bidirectional anatomical connectivity necessarily precludes such causal inference.

\section{Conclusion}

Regions of prefrontal cortex and the amygdala are responsible for the ability to regulate negative affect, findings that are bolstered here by the use of pupil diameter to verify the sustained active nature of the prescribed cognitive reappraisal strategies (increase, decrease) compared with an attention control condition. These data further indicate that there are individual differences in the ability to regulate, particularly in the ability to attenuate, negative affect as it unfolds over time. Individuals who show bigger reductions in the response of the amygdala when decreasing negative affect compared with the attention control are the same individuals who evidence greater responses in bilateral VMPFC for decreasing negative affect in response to aversive pictures compared with the attention control condition. Furthermore, linking the responses of the brain in a laboratory setting to the exigencies of daily life, higher VMPFC and lower amygdala signal during the downregulation of negative affect relative to the attention control condition, are associated with a more adaptive diurnal rhythm of circulating free cortisol secretion over the course of 1 week at home. It remains an issue for future studies to determine whether these associations generalize to other age groups because only older individuals were studied here. The neural mechanisms of emotion regulation may be different in older versus younger individuals. In summary, the pattern of findings herein suggests that functional coupling between the PFC and the amygdala enables effective regulation of negative emotion. Furthermore, the functioning of PFC-amygdala circuitry during regulation of negative affect predicts longer-term regulation of endocrine activity that may be important for health and well-being.

\section{References}

Abercrombie HC, Giese-Davis J, Sephton S, Epel ES, Turner-Cobb JM, Spiegel D (2004) Flattened cortisol rhythms in metastatic breast cancer patients. Psychoneuroendocrinology 29:1082-1092.

Baum A, Grunberg N (1995) Measurement of stress hormones. In: Measuring stress: a guide for health and social scientists (Cohen S, Kessler RC, Gordon LU, eds), pp 175-192. New York: Oxford UP.

Beauregard M, Levesque J, Bourgouin P (2001) Neural correlates of conscious self-regulation of emotion. J Neurosci 21:RC165(1-6).

Beck AT, Steer RA, Brown GK (1996) Manual for the Beck Depression Inventory. San Antonio, TX: The Psychological Corporation.

Burgess PS, Simons JS, Dumontheil I, Gilbert SJ (2005) The gateway hypothesis of rostral prefrontal cortex (area 10) function. In: Measuring the mind: speed, control, and age (Duncan J, Phillips L, McLeod P, eds), pp 217-248. Oxford: Oxford UP.

Burke HM, Davis MC, Otte C, Mohr DC (2005) Depression and cortisol 
responses to psychological stress: a meta-analysis. Psychoneuroendocrinology 30:846-856.

Center for the Study of Emotion and Attention (1999) The International Affective Picture System: digitized photographs. Gainesville, FL: Center for Research in Psychophysiology, University of Florida.

Diorio D, Viau V, Meaney MJ (1993) The role of the medial prefrontal cortex (cingulate gyrus) in the regulation of the hypothalamic-pituitaryadrenal responses to stress. J Neurosci 13:3839-3847.

Harenski CL, Hamann S (2006) Neural correlates of regulating negative emotions related to moral violations. NeuroImage 30:313-324.

Jackson DC, Malmstadt JR, Larson CL, Davidson RJ (2000) Suppression and enhancement of emotional responses to unpleasant pictures. Psychophysiology 37:515-522.

Jackson DC, Mueller CJ, Dolski IV, Dalton KM, Nitschke JB, Urry HL, Rosenkranz MA, Ryff CD, Singer BH, Davidson RJ (2003) Now you feel it, now you don't: frontal brain electrical asymmetry and individual differences in emotion regulation. Psychol Sci 14:612-617.

Janssens CJ, Helmond FA, Wiegant VM (1995) The effect of chronic stress on plasma cortisol concentrations in cyclic female pigs depends on the time of day. Domest Anim Endocrinol 12:167-177.

Kalisch R, Wiech K, Critchley HD, Seymour B, O'Doherty JP, Oakley DA, Allen P, Dolan RJ (2005) Anxiety reduction through detachment: subjective, physiological, and neural effects. J Cogn Neurosci 17:874-883.

Kim H, Somerville LH, Johnstone T, Alexander AL, Whalen PJ (2003) Inverse amygdala and medial prefrontal cortex responses to surprised faces. NeuroReport 14:2317-2322.

Kim Y, Pilkonis PA, Frank E, Thase ME, Reynolds CF (2002) Differential functioning of the Beck depression inventory in late-life patients: use of item response theory. Psychol Aging 17:379-391.

Kvaal K, Ulstein I, Nordhus IH, Engedal K (2005) The Spielberger StateTrait Anxiety Inventory (STAI): the state scale in detecting mental disorders in geriatric patients. Int J Geriatr Psychiatry 20:629-634.

Levesque J, Eugene F, Joanette Y, Paquette V, Mensour B, Beaudoin G, Leroux JM, Bourgouin P, Beauregard M (2003) Neural circuitry underlying voluntary suppression of sadness. Biol Psychiatry 53:502-510.

Levesque J, Joanette Y, Mensour B, Beaudoin G, Leroux JM, Bourgouin P, Beauregard M (2004) Neural basis of emotional self-regulation in childhood. Neuroscience 129:361-369.

Loftus GR, Masson ME (1994) Using confidence intervals in within-subject designs. Psychon Bull Rev 1:476-490.

Macaulay C, Battista M, Lebby PC, Mueller J (2003) Geriatric performance on the Neurobehavioral Cognitive Status Examination (Cognistat). What is normal? Arch Clin Neuropsychol 18:463-471.

Marti O, Gavalda A, Jolin T, Armario A (1993) Effect of regularity of exposure to chronic immobilization stress on the circadian pattern of pituitary adrenal hormones, growth hormone, and thyroid stimulating hormone in the adult male rat. Psychoneuroendocrinology 18:67-77.

McEwen BS (1999) Stress and hippocampal plasticity. Annu Rev Neurosci 22:105-122.

Milad MR, Quirk GJ (2002) Neurons in medial prefrontal cortex signal memory for fear extinction. Nature 420:70-74.

Ochsner KN, Bunge SA, Gross JJ, Gabrieli JDE (2002) Rethinking feelings: an fMRI study of the cognitive regulation of emotion. J Cogn Neurosci 14:1215-1229.

Ochsner KN, Ray RD, Cooper JC, Robertson ER, Chopra S, Gabrieli JD, Gross JJ (2004) For better or for worse: neural systems supporting the cogni- tive down- and up-regulation of negative emotion. NeuroImage 23:483-499.

Ohira H, Nomura M, Ichikawa N, Isowa T, Iidaka T, Sato A, Fukuyama S, Nakajima T, Yamada J (2006) Association of neural and physiological responses during voluntary emotion suppression. NeuroImage 29:721-733.

Phan KL, Fitzgerald DA, Nathan PJ, Moore GJ, Uhde TW, Tancer ME (2005) Neural substrates for voluntary suppression of negative affect: a functional magnetic resonance imaging study. Biol Psychiatry 57:210-219.

Phelps EA, Delgado MR, Nearing KI, LeDoux JE (2004) Extinction learning in humans: role of the amygdala and vmPFC. Neuron 43:897-905.

Polk DE, Cohen S, Doyle WJ, Skoner DP, Kirschbaum C (2005) State and trait affect as predictors of salivary cortisol in healthy adults. Psychoneuroendocrinology 30:261-272.

Preacher KJ, Hayes AF (2004) SPSS and SAS procedures for estimating indirect effects in simple mediation models. Behav Res Methods Instrum Comput 36:717-731.

Price JL (2005) Free will versus survival: brain systems that underlie intrinsic constraints on behavior. J Comp Neurol 493:132-139.

Quirk GJ, Likhtik E, Pelletier JG, Pare D (2003) Stimulation of medial prefrontal cortex decreases the responsiveness of central amygdala output neurons. J Neurosci 23:8800-8807.

Ramnani N, Owen AM (2004) Anterior prefrontal cortex: insights into function from anatomy and neuroimaging. Nat Rev Neurosci 5:184-194.

Ranjit N, Young EA, Kaplan GA (2005) Material hardship alters the diurnal rhythm of salivary cortisol. Int J Epidemiol 34:1138-1143.

Rosenkranz MA, Jackson DC, Dalton KM, Dolski I, Ryff CD, Singer BH, Muller D, Kalin NH, Davidson RJ (2003) Affective style and in vivo immune response: neurobehavioral mechanisms. Proc Natl Acad Sci USA 100:11148-11152.

Sapolsky RM (2003) Stress and plasticity in the limbic system. Neurochem Res 28:1735-1742.

Schaefer SM, Jackson DC, Davidson RJ, Aguirre GK, Kimberg DY, Thompson-Schill SL (2002) Modulation of amygdalar activity by the conscious regulation of negative emotion. J Cogn Neurosci 14:913-921.

Sephton SE, Sapolsky RM, Kraemer HC, Spiegel D (2000) Diurnal cortisol rhythm as a predictor of breast cancer survival. J Natl Cancer Inst 92:994-1000.

Spielberger CD, Gorsuch RL, Lushene RE, Vagg PR, Jacobs GA (1983) Manual for the state-trait anxiety inventory. Palo Alto, CA: Consulting Psychologists.

Sullivan GM, Apergis J, Bush DE, Johnson LR, Hou M, LeDoux JE (2004) Lesions in the bed nucleus of the stria terminalis disrupt corticosterone and freezing responses elicited by a contextual but not by a specific cueconditioned fear stimulus. Neuroscience 128:7-14.

Sullivan RM, Gratton A (1999) Lateralized effects of medial prefrontal cortex lesions on neuroendocrine and autonomic stress responses in rats. J Neurosci 19:2834-2840.

Urry HL, Nitschke JB, Dolski I, Jackson DC, Dalton KM, Mueller CJ, Rosenkranz MA, Ryff CD, Singer BH, Davidson RJ (2004) Making a life worth living: neural correlates of well-being. Psychol Sci 15:367-372.

Yehuda R, Yang RK, Buchsbaum MS, Golier JA (2006) Alterations in cortisol negative feedback inhibition as examined using the ACTH response to cortisol administration in PTSD. Psychoneuroendocrinology 31:447-451. 